\title{
VALOR PROGNÓSTICO DA FIBRONECTINA PLASMÁTICA E DA CLASSIFICAÇÃO DE CHILD -PUGH NA CIRROSE HEPÁTICA ALCOÓLICA. ESTUDO COMPARATIVO
}

\author{
Edison Roberto Parise*, flávio Hugo Parisi, Marilisa de Moraes B. Leite-Mór, Marie Doki Nogueira \\ Trabalho realizado na disciplina de Gastroenterologia da Universidade Federal \\ de São Paulo, Escola Paulista de Medicina, São Paulo, SP.
}

RESUMO - OBjetivos. Avaliar o valor prognóstico da fibronectina plasmática (FN), comparativamente à classificação numérica de Child-Pugh e os parâmetros bioquímicos que a compõem, no acompanhamento prospectivo de portadores de cirrose alcoólica durante 18 meses.

Métodos. Incluídos 50 pacientes com cirrose alcoólica, diagnosticada por biópsia ou critérios clínico-bioquímicos, excluídos aqueles com hepatocarcinoma ou hemorragia digestiva, infecção ou ingestão alcoólica continuada nos últimos 30 dias. A idade média do grupo foi $51,3 \pm 12,6$ anos, $72 \%$ deles do sexo masculino e classificados 17 como Child-Pugh A, 18 como B e 15 como C. Os valores das bilirrubinas foram dosados pelo método automatizado, eletroforese de proteínas em acetato de celulose e o tempo de protrombina pelo método de Quick. A FN plasmática foi dosada por imunodifusão radial, com anticorpos contra FN humana em géis de agarose a $1 \%$.

Resultados. Um paciente foi excluído por óbito de causa não natural e 12 foram a óbito por doença hepática. Os melhores preditores de óbito foram a pontuação de ChildPugh [escore $>10$, risco relativo (RR) de II,33) e os valores de bilirrubina $(>2,5 \mathrm{mg} / \mathrm{dL}, \mathrm{RR}=9,47)$. A concentração de $\mathrm{FN}$ foi significantemente maior nos sobreviventes que naqueles que foram a óbito $(185 \pm 66 \mathrm{mg} / \mathrm{L} \times 13 \mathrm{I} \pm 38 \mathrm{mg} / \mathrm{L}, \mathrm{p}<0,0 \mathrm{I})$, com $R R=6,59$ para $\mathrm{FN}<165 \mathrm{mg} / \mathrm{L}$. Valores de $\mathrm{FN}$ acima desse valor de corte, entretanto, foram os melhores indicadores de sobrevida desde que $96,5 \%$ desses 29 pacientes estavam vivos ao final de 18 meses de seguimento.

ConcLusão. Embora apresente menor acurácia em predizer 0 risco de óbito desses pacientes, valores de FN plasmática $>165 \mathrm{mg} / \mathrm{L}$ foram melhores indicadores de sobrevida que a classificação de Child-Pugh e seus parâmetros bioquímicos isolados.

Unitermos: Fibronectina plasmática. Classificação Child-Pugh. Cirrose alcoólica. Sobrevida na cirrose.

\section{INTRODUÇÃO}

A fibronectina (FN) é uma glicoproteína encontrada no sangue circulante (forma solúvel) ou depositada nos tecidos, inclusive como constituinte da membrana basal (forma insolúvel ou tecidual). Esta glicoproteína tem sido associada a vários processos biológicos como adesão e diferenciação celular; reparação de tecidos e fagocitose de bactérias, complexos antígeno-anticorpos, servindo como substrato para enzimas fibrinolíticas e da coagulação ${ }^{1-4}$.

Vários tipos celulares podem sintetizar fibronectina. No fígado, as células parenquimatosas e, praticamente todas células sinusoidais, como células de Kupffer, células

\footnotetext{
*Correspondência:

Disciplina de Gastroenterologia

Universidade Federal de São Paulo

Rua Botucatu, $740-2^{\circ}$ andar - Cep: 04023-090

São Paulo - SP - Fax-Fone (11) 5549.1711

E-mail: parise@gastro.epm.br
}

endoteliais e estelares são capazes de sintetizar essa glicoproteína ${ }^{1,5-7}$. Entretanto, a maioria dos trabalhos sugerem que a maior parte, senão toda a fibronectina circulante, seja produzida pelos hepatócitos ${ }^{5-9}$. Essa produção pelas células parenquimatosas seria responsável pela observada correlaçãao entre os níveis de fibronectina circulante e o grau de disfunção hepatocelular, tanto em hepatopatias agudas como crônicas ${ }^{10-13}$. Pelo menos um estudo demonstrou que os valores séricos da fibronectina pode ter valor prognóstico na determinação do risco de óbito ou de sobrevida de portadores de cirrose hepática alcoólical ${ }^{14}$. Entretanto, resta saber qual a contribuição efetiva que a dosagem dos níveis circulantes dessa glicoproteína poderia ter para o prognóstico desses pacientes, quando comparadas a outras determinações mais tradicionalmente utilizadas para esse fim. De fato, a atividade de protrombina, a bilirrubina e a classificação de Child-Pugh são avaliações já incorporadas na prática médica, de fácil execução e longo tempo de uso além de inequívoco valor prognóstico na cirrose hepátical ${ }^{15-17}$.

Nesse trabalho comparamos o valor prognóstico da fibronectina plasmática com parâmetros clínicos e bioquímicos freqüentemente utilizados na clínica médica para predizer o óbito em portadores de cirrose hepática de etiologia alcoólica, acompanhados prospectivamente por período mínimo de 18 meses.

\section{Métodos}

Foram estudados 50 pacientes do ambulatório de doenças hepáticas da disciplina de Gastroenterologia da Universidade Federal de São Paulo - Escola Paulista de Medicina, com idade média de $51,3 \pm 12,6$ anos (média \pm desvio padrão). Os pacientes foram selecionados a partir do diagnóstico de cirrose hepática de etiologia alcoólica pela ingestão de pelo menos $80 \mathrm{~g}$ de etanol ao dia, por tempo mínimo de 10 anos. 0 diagnóstico de cirrose hepática foi feito 
por biópsia hepática em $62 \%$ dos casos e nos demais através dos dados clínicos e laboratoriais $^{18}$, além de exame endoscópico e ultra-sonográfico. Foram excluídos pacientes com idade superior a 65 anos; com infecção sistêmica ou localizada; apresentando hepatocarcinoma ou outras doenças crônicas associadas. Todos os pacientes apresentavam varizes esofagogástricas à endoscopia digestiva e negavam a ingestão de bebidas alcoólicas nos 30 dias que antecederam a realização dos exames de base. De acordo com os critérios de Child, modificados por Pugh et al. ${ }^{19}$, os pacientes foram classificados funcionalmente em três grupos distintos: Child $A=17$ pacientes com idade média de 51,8 anos; Child $B=18$ pacientes com idade média de 52,3 anos e Child $\mathrm{C}=15$ pacientes com idade média de 59,5 anos. Desses pacientes, 20 foram classificados como compensados e 30 como descompensados, de acordo com a presença ou não de ascite, icterícia e/ou encefalopatia. Os pacientes assim selecionados foram submetidos à determinação de provas bioquímicas rotineiramente efetuadas, como: bilirrubinas, fosfatase alcalina, gamaglutamil transpeptidase (GGT), AST, ALT , por método automatizado, eletroforese de proteínas em acetato de celulose e tempo e atividade de protrombina (TAP) pelo método de Quick. Marcadores virais para hepatite $\mathrm{B}(\mathrm{HBsAg}$, anti-HBc) e C (anti-HCV) foram determinados pelo método de ELISA, com kits da Abbott Laboratórios. A dosagem de fibronectina no plasma foi feita pela técnica de imunodifusão radial simples usando anticorpos contra fibronectina humana (Sigma Chemical Co., Mo, USA) em geis de agarose a $1 \%$. As placas de agarose foram preparadas utilizando o anticorpo antifibronectina na concentração de $1 / 100$. Diluições de um padrão de proteínas plasmáticas humanas contendo concentração conhecida de fibronectina $(250 \mathrm{mgldl}$, Behring, Marburg, Germany) foram utilizadas para obtenção da curva de referência. $\mathrm{O}$ sangue para determinação da fibronectina foi colhido em veia periférica com paciente em jejum de pelo menos 8 horas, em frasco contendo citrato de sódio a 3,8\%.

Os pacientes foram acompanhados em regime ambulatorial por período médio de 18 meses, com retornos regulares mensais nos

\begin{tabular}{|c|c|c|c|}
\hline & $\begin{array}{l}\text { Óbitos } \\
(n=12)\end{array}$ & $\begin{array}{c}\text { Sobreviventes } \\
(\mathrm{n}=37)\end{array}$ & $\mathrm{p}$ \\
\hline $\begin{array}{c}\text { Idade } \\
\text { Albumina } \\
\text { AP(\%) } \\
\text { Bilirrubinas (mg/dL) } \\
\text { Fibronectina(mg/dL) }\end{array}$ & $\begin{array}{c}44,1 \pm 16 \\
3,12 \pm 1,20 * \\
36 \pm 19 * \\
6,4 \pm 2,0 * \\
131 \pm 38 *\end{array}$ & $\begin{array}{c}50,7 \pm 11,7 \\
3,38 \pm 1,67 \\
68 \pm 20 \\
1,7 \pm 1,1 \\
185 \pm 66\end{array}$ & $\begin{array}{l}0,10 \\
<0,05 \\
<0,01 \\
<0,01 \\
<0,01\end{array}$ \\
\hline
\end{tabular}

(*) valores estatisticamente significantes em relação aos pacientes que sobreviveram. AP = atividade de protrombina(*) $p<0,05$, teste " $t$ " de Student

Figura I - Sobrevida acumulada em portadores de cirrose hepática alcoólica em 18 meses de seguimento, subdivididos de acordo com concentração inicial da fibronectina plasmática

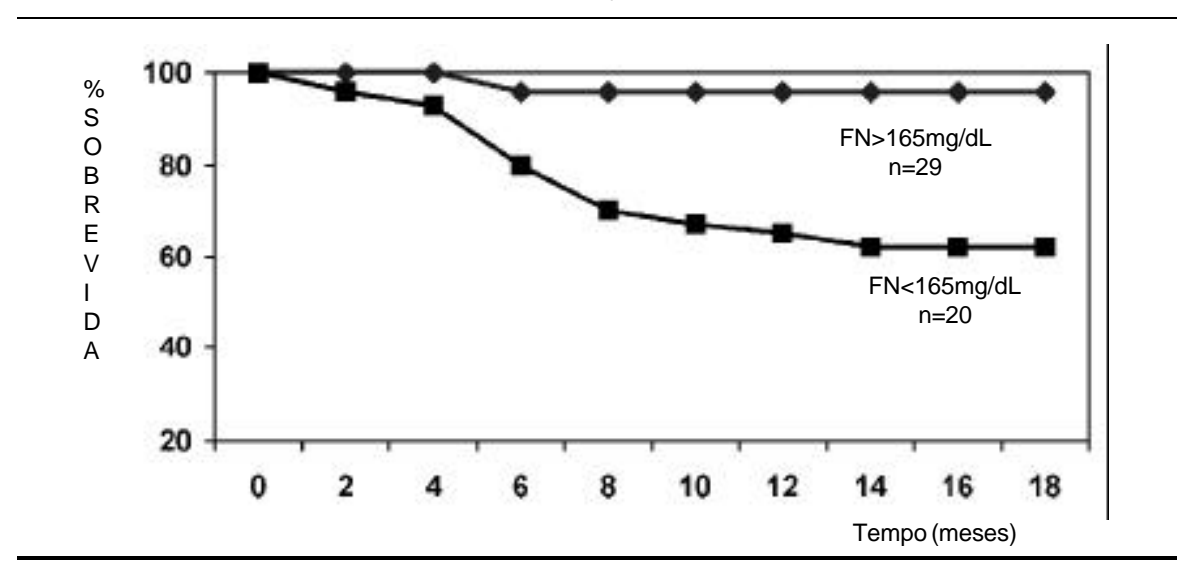

primeiros três meses e, depois, a cada três meses, observando-se a ocorrência ou não de óbito por descompensação ou complicação decorrente da doença hepática ou de complicação paralela.

\section{Método Estatístico}

Para comparação das médias das variáveis estudadas empregou-se o teste " $\mathrm{t}$ " de Student. A avaliação do poder discriminativo de cada variável considerada foi feita a partir de indicadores de sensibilidade, especificidade, acurácia, valor preditivo positivo e valor preditivo negativo. Para o cálculo do risco relativo empregou-se o teste do Qui-quadrado. Os cálculos foram feitos por microcomputador, utilizando-se os programas estatísticos Epiinfo. Em todos os testes fixou-se em 0,05 ou $5 \%$ o nível de rejeição da hipótese de nulidade, assinalando-se com um asterisco $(*)$ os valores significantes do cálculo estatístico ${ }^{20,21}$

\section{Resultados}

Todos pacientes foram acompanhados até completar 18 meses. Nesse período, I3 pacientes foram a óbito, sendo um por causa não natural e os 12 restantes em decorrência de complicações da doença hepática, perfazendo um total de $24,5 \%$ (12/49) dos pacientes acompanhados. Os pacientes que foram a óbito apresentavam função hepática significantemente mais comprometida do que os pacientes que sobreviveram ao período de acompanhamento, conforme pode ser visto pelos valores séricos de albumina, bilirrubinas totais e tempo de protrombina (Tabela I). A média dos valores de fibronectina foi estatisticamente menor no grupo que foi a óbito que no grupo de sobreviventes ao final do período de seguimento (Tabela I).

Quando os pacientes foram separados em dois grupos, de acordo com a concentração 
Tabela 2 - Avaliação do risco relativo de óbito do paciente para os parâmetros avaliados, em intervalo de confiança de $95 \%$, ao final de 18 meses de seguimento

\begin{tabular}{|c|c|c|c|}
\hline & $\begin{array}{l}\text { Pacientes que } \\
\text { foram a óbito }\end{array}$ & $\begin{array}{l}\text { Pacientes que } \\
\text { sobreviveram }\end{array}$ & $\begin{array}{c}\text { Risco } \\
\text { relativo }\end{array}$ \\
\hline $\begin{array}{l}\text { Child-Pugh }>10 \text { pontos } \\
\text { Bilirrubinas }>2,5 \mathrm{mg} \% \\
\text { Fibronectina }<165 \mathrm{mg} / \mathrm{L} \\
\text { AP }<50 \% \\
\text { AscitePresente }\end{array}$ & $\begin{array}{l}10 / 12(83 \%) \\
10 / 12(83 \%) \\
11 / 12(92 \%) \\
09 / 12(75 \%) \\
07 / 12(58 \%)\end{array}$ & $\begin{array}{l}05 / 37(13,5 \%) \\
07 / 37(18,9 \%) \\
09 / 37(24,3 \%) \\
07 / 37(18,9 \%) \\
07 / 37(18,9 \%)\end{array}$ & $\begin{array}{r}11,33 \\
9,47 \\
6,59 \\
6,19 \\
3,32\end{array}$ \\
\hline
\end{tabular}

Tabela 3 - Poder discriminativo (em percentual) dos valores de fibronectina plasmática em comparação com a bilirrubina sérica e a classificação de Child-Pugh, expressa em pontos

\begin{tabular}{|c|c|c|c|c|c|}
\hline & Sensibilidade & Especificidade & VPP & VPN & Acurácia \\
\hline Child-Pugh & 83 & 86,5 & $\overline{66,7}$ & $\overline{94}$ & 85,7 \\
\hline Bilirrubinas & 83 & 81 & 59 & 93,7 & 81,6 \\
\hline Fibronectina & 92 & 76 & 55 & 96,5 & 79,6 \\
\hline
\end{tabular}

VPP = valor preditivo positivo; VPN = valor preditivo negativo

sérica de fibronectina (> ou $<$ de $165 \mathrm{mg} / \mathrm{L}$ ), observa-se evidente pior sobrevida nos pacientes com valores mais abaixo do limite de corte preconizado pela literatura ${ }^{14}$.

Para o estudo comparativo entre o valor prognóstico da fibronectina e os parâmetros clínicos e bioquímicos preditivos de sobrevida nesses portadores de cirrose hepática, avaliamos o risco relativo de óbito do paciente em função dos valores de cada uma das variáveis estudadas, empregando o teste do Qui-quadrado (Tabela 2). Nessa tabela observa-se que para um intervalo de confiança de $95 \%$, os valores séricos das bilirrubinas e, principalmente, a classificação de Child-Turcotte modificada por Pugh foram superiores à dosagem da concentração de fibronectina como fator de risco para óbito nesses pacientes. Assim, pacientes com Child-Pugh superior a 10 pontos apresentavam risco relativo de óbito mais de II vezes superior aos pacientes com Child numérico inferior a esse limite de corte, enquanto para a fibronectina plasmática, esse risco relativo foi de 6,5 vezes para o limite de corte preconizado na literatura de $165 \mathrm{mg} / \mathrm{L}^{14}$.

Comparando-se a acurácia diagnóstica da fibronectina em relaçãao apenas à classificação de Child-Pugh expressa em pontos e aos valores de bilirrubinas, observa-se (Tabela 3) que a fibronectina teve sensibilidade e valor preditivo negativo superiores aos observados para os outros dois parâmetros.

\section{Discussão}

Modificações da concentração plasmática da fibronectina têm sido observadas em várias condições clínicas, como septicemias, coagulação intra-vascular disseminada, hepatites fulminantes, neoplasias e hepatopatias crônicas 2,10,13,23.

A relação entre concentração sérica dessa glicoproteína e doença hepática crônica tem sido creditada à sua síntese preferencial pelas células hepáticas ${ }^{7-9}$. Embora alguns autores tenham sugerido uma relação com o grau de hipertensão portal e de hiperesplenismo ${ }^{22,23}$, a maioria das evidências indica que a redução nos níveis plasmáticos da fibronectina nos pacientes com hepatopatia crônica esteja mais diretamente relacionada à função hepática desses pacientes ${ }^{2}, 11,14,24$. Entretanto, deve-se salientar que a função hepática não deve ser o único fator a alterar os níveis circulantes da glicoproteína nesses pacientes, uma vez que essa correlação é claramente observada somente em cirróticos com comprometimento funcional mais acentuado ${ }^{2,13}$.

A busca de parâmetros que possam predizer a evolução dos hepatopatas crônicos tornou-se ainda mais crucial com o advento do transplante hepático, uma vez que sua indicação precoce melhora o resultado e reduz os custos do procedimento. Apesar dos inúmeros testes funcionais, dosagens bioquímicas e marcadores teciduais propostos, a classificação de Child-Pugh tem se mantido como padrão ouro na avaliação prognóstica desses pacientes. Mesmo incluindo indicadores clínicos sujeitos à subjetividade e parâmetros laboratoriais sem especificidade absoluta, expressa com boa acurácia o grau de comprometimento funcional hepático existente ${ }^{13}$. É considerado um método simples e confiável, sendo acessível a qualquer centro de investigação clínica ${ }^{25}$. Além disso, a classificação funcional de Child-Pugh, especialmente quando expressa em sua forma de pontuação, é considerada um indicador de grande valor prognóstico em relação à expectativa de sobrevida de pacientes hepatopatas crôni$\cos ^{15,17,26}$. Mesmo com a proposta de um novo índice prognóstico denominado de modelo para doença hepática crônica em estágio final (MELD, na língua inglesa), a classificação de Child-Pugh ainda persiste como importante índice prognóstico 27,28 .

Assim, para testar o valor prognóstico da fibronectina resolvemos confrontar seus resultados com os valores numéricos dessa classificação ou mesmo com os seus parâmetros isoladamente, desde que tanto os níveis de bilirrubinas, albumina e a atividade de protrombina, como a presença ou ausência de encefalopatia e ascite, são considerados parâmetros de grande sensibilidade prognóstica na cirrose hepática 15,17,26.

No período de acompanhamento observaram-se 12 óbitos decorrentes de complicações da doença hepática, especialmente hemorragia digestiva e infecções. Quase a totalidade dos óbitos ocorreram dentro de 12 meses de seguimento, sendo maior nos três primeiros meses. Conforme demonstrado nos resultados, o risco relativo de óbito para a determinação da fibronectina plasmática foi inferior à classificação de Child-Pugh e à dosagem das bilirrubinas plasmáticas. Além disso, a dosagem da glicoproteína tem contra si o fato de não ser uma técnica encontrada na rotina dos laboratórios e, assim, representa uma metodologia mais trabalhosa e onerosa que a dosagem da albumina, bilirrubinas e 0 tempo de protrombina.

Entretanto, ao analisarmos com maior cuidado a acurácia diagnóstica da fibronectina, observamos que ela apresenta sensibilidade e valor preditivo negativo superiores às demais determinações. Entretanto, embora 92\% dos 
Parise ER et al.

pacientes que foram a óbito apresentassem valores inferiores a $165 \mathrm{mg} / \mathrm{L}$, eles perfaziam apenas $55 \%$ dos pacientes abaixo desse valor. Isso reduziu o valor da glicoproteína como índice de risco para óbito. Por outro lado, 28 dos 29 pacientes $(96,5 \%)$ que apresentaram valores iniciais de fibronectina acima desse valor de corte de $165 \mathrm{mg} / \mathrm{L}$, sobreviveram. Esses números caracterizaram esse parâmetro como o melhor índice de sobrevida entre os pacientes estudados, confirmando o trabalho de Naveau et al. ${ }^{14}$ que também demonstraram ter sido a fibronectina sérica a variável de maior valor prognóstico numa análise multivariada.

\section{SUMMARY}

\section{Prognostic value of plasmatic FIBRONECTIN AND ChILD-Pugh CLASSIFI- CATION IN ALCOHOLIC CIRRHOTIC PATIENTS. A comparative study.}

BACKGROUND. To assess the prognostic value of plasmatic fibronectin (FN), compared to numeric Child-Pugh classification and its biochemical parameters in patients with alcoholic cirrhosis followed prospectively during a 18 months-period.

MetHOds. Fifty patients with the diagnosis of cirrhosis by hepatic biopsy or clinical and biochemical criteria, were included in the study after the exclusion of hepatocarcinoma and $\mathrm{Gl}$ bleeding, infection or continous alcohol ingestion in the last 30 days. The mean age was $51.3 \pm 12.6$ years, being $72 \%$ males and 17 of them were classified as Child-Pugh A, 18 as B and 15 as $C$. Serum bilirrubin concentration was measured in autoanalyzer, protein electrophoresis was performed on cellulose acetate and prothrombin time by the Quick test. Plasmatic FN was assessed by radial immunodifusion with anti-human FN in $1 \%$ agarose gel slabs.

RESULTS. One patient was excluded because no natural death and 12 died owing to hepatic disease. The numeric Child-Pugh [score $>10$, Relative Risk $(R R)=11.33]$ and total bilirrubins $(>2.5 \mathrm{mg} / \mathrm{dL}, R R=9.47$ ) were the best predictors of death. Mean plasmatic FN concentration was significantly higher among those who survived when compared with those who died $(185 \pm 66 \mathrm{mg} / \mathrm{L} \times 131 \pm 38 \mathrm{mg} / \mathrm{L}, p<0,01)$, with a $R R=6.59$, for $F N<165 \mathrm{mg} / \mathrm{L}$. Higher levels of $F N$, on the other hand, were the best variable to predict survival, since $96 \%$ of these 29 patients were alive at the end of follow-up.

Conclusion. Although having less accuracy in predicting the risk of death of these patients, plasmatic FN $>165 \mathrm{mg} / \mathrm{L}$ was better predictor of survival than Child-Pugh score or any one of its biochemical parameters. [Rev Assoc Med Bras 2004; 50(I): 37-40]

KEY wORDS: Plasmatic fibronectin. Child-Pugh classification. Alcoholic cirrhosis and survival in cirrhosis.

\section{RefERÊNCIAS}

I. Alitalo K, Hovi T, Vaheri A. Fibronectin is produced by human macrophages. J Exp Med 1980; | $51: 602-13$
2. Matsuda A. A distribution of fibronectin in plasma and liver disease. Clin Chim Acta 1982 | | 8:191-9.

3. Mosesson MW, Amrani DL. The structure and biologic activities of plasma fibronectin. Blood | 980; 56: |45-58.

4. Stenman S, Vaheri A. Distribution of a major connective tissue fibronectin, in normal human tissues. J Exp Med 1978; |47: 1054-64

5. Neubauer K, Kruger M, Quondamatteo F, Knittel T, Saile B, Ramadori G. Transforming growth factor-beta I stimulates the synthesis of basement membrane proteins laminin collagen type IV and entactin in rat liver sinusoidal cells. J Hepatol 1999, 31: 692-702.

6. Parise ER, Summerfield JÁ, Wiedmann KH, Hahn E, Doenhoff MJ. Basement membrane proteins and type III procolagen in murine schistosomiasis. Trans R Soc Trop Med Hyg 1985: 79: 663-70.

7. Voss B, Allam S, Rauterberg J, Ullrich K, Gieselmann V, Von Figura K. Primary cultures of rat hepatocytes synthesize fibronectin. Biochem Biophys Res Commun. 1979, 90: I348-54.

8. Tamkun JW, Hynes RO. Plasma fibronectin is synthesized by hepatocytes. J Biol Chem 1983 258: $464 \mid-7$.

9. Xu G, Niki T, Virtanen I, Rogiers V, Debleser P, Geerts A. Gene expression and synthesis of fibronectin isoforms in rat hepatic stellate cells. Comparison with liver parenchymal cells and skin fibroblasts. J Pathol 1997, 183: 90-8.

10. Anand AC, Irshad M, Acharya SK, Gandhi SM, loshi YK, Tandon BN. Fibronectin in acute and subacute hepatic failure. J Clin Gastroenterol | 989; | |:3|4-9.

Artigo recebido: 21/05/02 Aceito para publicação: $31 / 07 / 03$ 